\title{
Optoelectronic Chaotic Circuits
}

\author{
M.P. Hanias, H.E. Nistazakis and G.S. Tombras \\ Department of Electronics, Computers, Telecommunications and Control, \\ Faculty of Physics, National and Kapodistrian University of Athens, Athens, 15784
}

Greece

\section{Introduction}

Deterministic chaotic systems exhibit great efficiency, since they react sensitively to small perturbations and thus it is feasible to be controlled and produce signals with small amounts of energy. Moreover, these systems, have the potential for great informationbearing capacity, since the complex signals and the variety of the produced states offers more possibilities for compact conventional encoding of information signals. One of the major application of deterministic chaotic systems is the field of optical communications (Argyris et al., 2005). The significant advantage chaotic optoelectronic systems is that there are not the restrictions of the standard spectra of sinusoidal frequency bands and thus, the number of available communication channels could be larger than the ones of linear systems and the only limit by the ability of receivers to distinguish between different chaotic states (Rizomiliotis et al., 2010). Due to the significant benefits which can offer this scientific area the research direction of dynamical chaos is gradually moving towards practical applications and thus, there is a growing interest for chaotic signal generation sources. In this frame, various circuits have been proposed, among which active chaotic oscillators are preferably considered due to their relative simplicity and energy efficiency. Such a circuit may be externally triggered, i.e. externally driven to chaotic oscillation and it can typically consist of only one active and a few passive components (Argyris et al., 2010). In this respect, it is reasonable to expect that optoelectronic elements, such as a light emitting diode (LED) and optocoupler devices, will provide for the nonlinear characteristics required towards chaos.

The optoelectronic circuits of the chaotic oscillators must be as simply as they can so in order to be possible to synchronize them. Chaos, in this case, is created by the way that the components of the circuits are connected in order to operate in a non linear manner and not by the specific characteristic that each item has (Romeira et al., 2009). The advantage of such an operation is that in this way we avoid the inherent and unpredictable instabilities in the operation of optical devices (i.e. impurities concentration, rate of carrier recombination, crystal defects, e.t.c.).

In this chapter, we present simple chaotic circuits that can be utilized as chaotic signal generation, sources, main transmitters, as well as nonlinear observer-based main receivers for chaos based communication systems. These circuits are classified as Resistor-InductorLED optoelectronic chaotic circuits, single optocoupling device and optoelectronic Simulation of the Duffing-Holmes Equation. 
The operational characteristics of these circuits are studied, by simulations, using circuit simulation software (e.g. Multisim). Following that, we investigate the influence of various circuit parameters to the complexity of the so generated strange attractors. From the obtained -calculated and recorded-time series, we estimate, with non-linear analysis, the invariant parameters, as correlation, embedding dimension, Kolmogorov entropy and Lyapunov exponents, of the corresponding strange attractors as function of the control parameters.

\section{RL-LED optoelectronic circuits}

\subsection{Circuit's description}

A non autonomous chaotic circuit driven RL-LED circuit (Hanias et al., 2008) is shown in Figure 1. It consists of a series connection of an ac-voltage source, a linear resistor $R_{1}$, a linear inductor $L_{1}$ and a typical LED. The value of $R_{1} 100 \Omega$ and inside the circuits has been placed in series with the LED. In the circuit's input has been applied a sinusoidal voltage with amplitude $V_{1}$ as applied through an inductor $L_{1}$ with value $47 \mathrm{mH}$. The simulated circuit operation is monitored by checking the voltage value across resistor $R_{1}$. In Figure 2 is shown the, obtained by the simulation, chaotic time series at the output for input signal's amplitude $V_{1, r m s}=7 \mathrm{~V}$ and frequency $f=10 \mathrm{KHz}$.

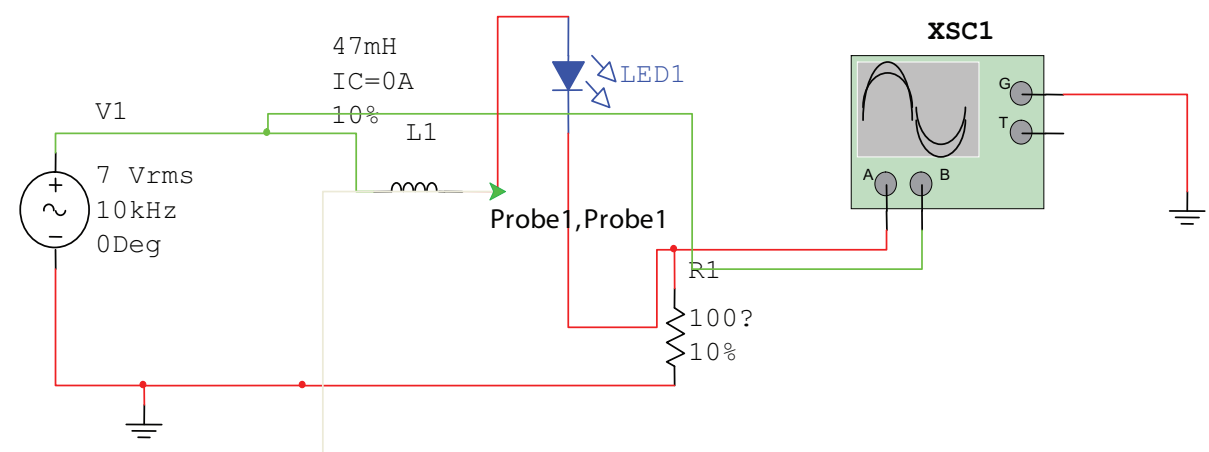

\footnotetext{
$\mathrm{V}: 2.38 \mathrm{~V}$

$\mathrm{V}(\mathrm{p}-\mathrm{p}): 16.5 \mathrm{~V}$

$\mathrm{V}(\mathrm{rms}): 7.72 \mathrm{~V}$

$\mathrm{V}(\mathrm{dc}):-13.7 \mathrm{mV}$

I: -248 uA

I(p-p): $4.26 \mathrm{~mA}$

I(rms): $2.58 \mathrm{~mA}$

I(dc): $1.78 \mathrm{~mA}$

Freq.: $19.7 \mathrm{kHz}$
}

Fig. 1. RL-LED chaotic circuit in Multisim circuits simulation software 


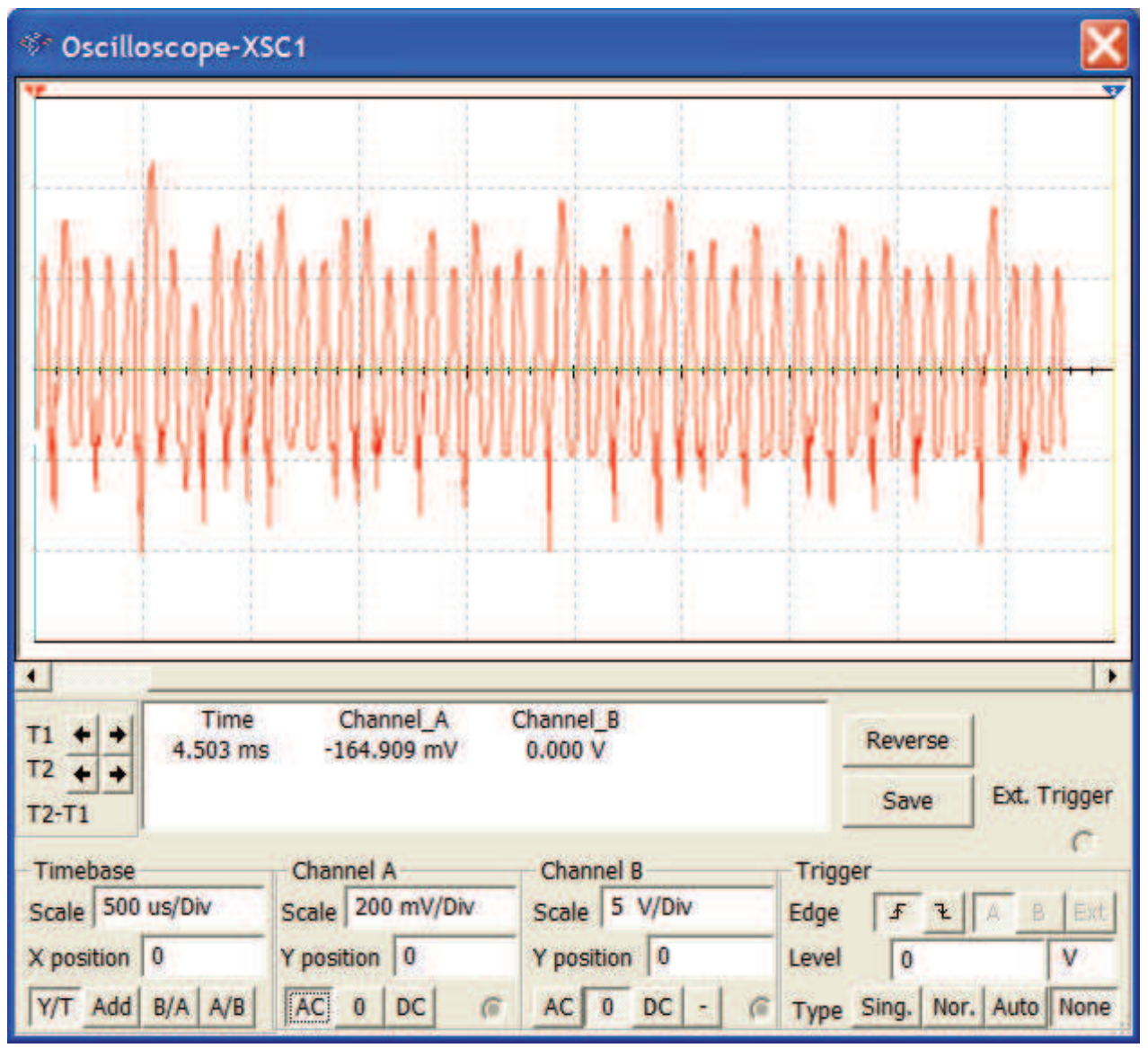

Fig. 2. Chaotic signal $V=V(t)$ across resistor $R_{1}$ for the RL-LED circuit of Figure 1

\subsection{Non-linear analysis}

Next, we proceed to the analysis of the obtained chaotic time series following the method proposed by Grassberger and Procaccia (Grassberger \& Procaccia, 1983) and successfully applied in similar cases (Hanias \& Anagnostopoulos, 1993). Additionally, according the Takens theory (Takens, 1981), the measured time series can be used to reconstruct the original phase space. At first, we calculate the correlation integral $C(r)$ for the simulated output signal, for $\lim (r)=0$ and $\lim (N)=\infty$ ( $N$ represents the number of the corresponding time series points), as defined by Kantz and Schreiber (Kanz \& Schreiber, 1997):

$$
C(r)=\frac{1}{N_{\text {pairs }}} \sum_{\substack{l=1, j=l+W}}^{N} H\left(r-\left\|\vec{X}_{l}-\vec{X}_{j}\right\|\right)
$$

where $W$ is the Theiler window (Kanz and Schreiber, 1997), $H$ is the Heaviside function, and $N_{\text {pairs }}$ is given as: 


$$
N_{\text {pairs }}=\frac{2}{(N-m+1)(N-m+W+1)}
$$

with $m$ being the embedding dimension. It is clear that the summation in (1) counts the number of pairs $\left(\vec{X}_{l}, \vec{X}_{j}\right)$ for which the distance, i.e. the Euclidean norm $\left\|\vec{X}_{l}-\vec{X}_{j}\right\|$ is less than $r$ in an $m$ dimensional Euclidean space. Here, the number of the experimental points is $N=10896$, while, considering the $m$ dimensional space, each vector $\vec{X}_{l}$ will be given as,

$$
\vec{X}_{l}=\left\{V\left(t_{i}\right), V\left(t_{i}+\tau\right), V\left(t_{i}+2 \tau\right), \ldots, V\left(t_{i}+(m-1) \tau\right)\right\}
$$

and represents a point of the $m$ dimensional phase space in which the attractor is embedded each time. In equation (3), $\tau$ is the delay time factor determined by the first minimum of the mutual information function $I(\tau)$ and defined as $\tau=\ell \Delta t$ with $\ell=1,2, \ldots, \mathrm{N}$ where $\Delta t=6.25 \mu \mathrm{s}$ is the sample rate. As shown in Figure 3, in our case, the mutual information function $I(\tau)$ exhibits a local minimum at $\tau=5$ time steps and thus we consider this value as the optimum one.

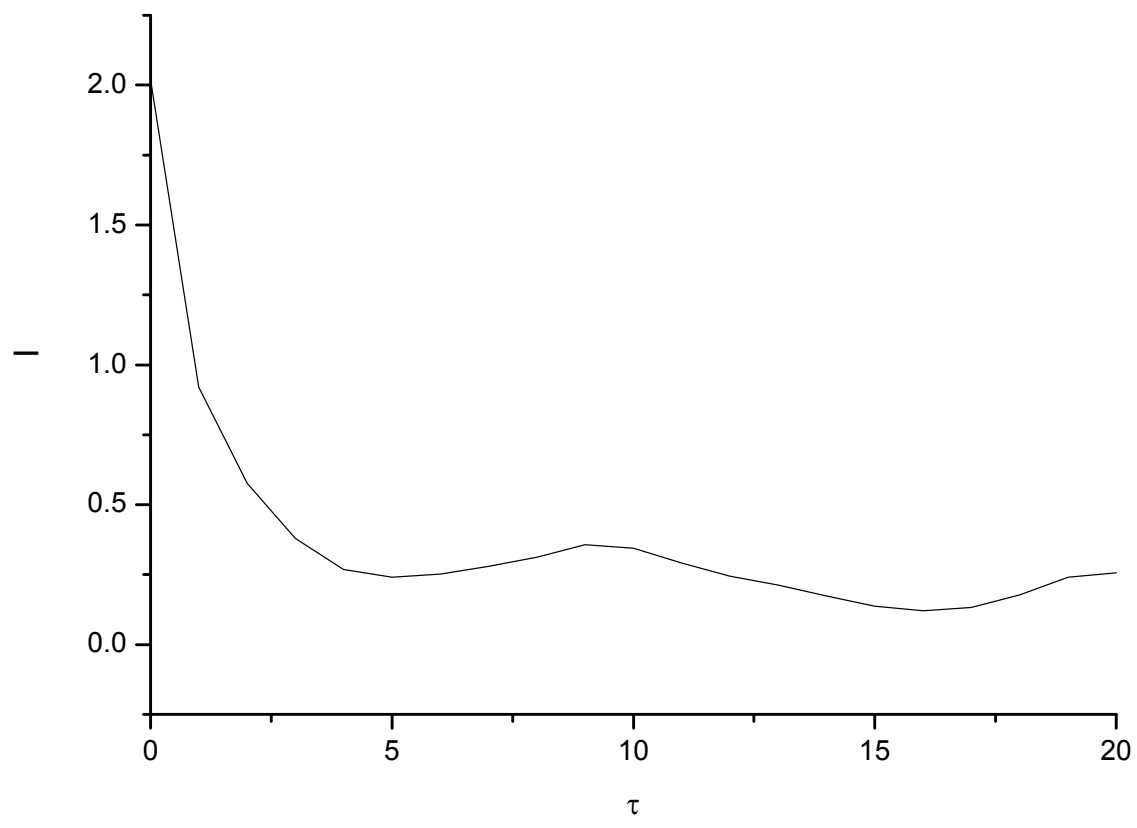

Fig. 3. Average mutual information $I(\tau)$ versus delay time $\tau$

Next, we investigate the parameter $W$ which is the Theiler window. As Theiler pointed out if temporally correlated points are not neglected, spuriously low dimension estimate may be 
obtained (Stelter \& Pfingsten, 1991). However, since there is no concrete rule of how to choose $W$, it may take the first zero-crossing value of the correlation function $C R(\tau)$ (Kanz \& Schreiber, 1997), as suggested by Kantz and Schreiber (Kanz \& Schreiber, 1997). This means that we can use the correlation length as a starting value for $W$. As shown in Figure 4, the correlation length is equal to 5 and thus, $W=5$ time lags. Figure 4 also depicts a strong correlation between the data indicating the way past states affect the system's current state. Hence, we can use these values for phase space reconstruction.

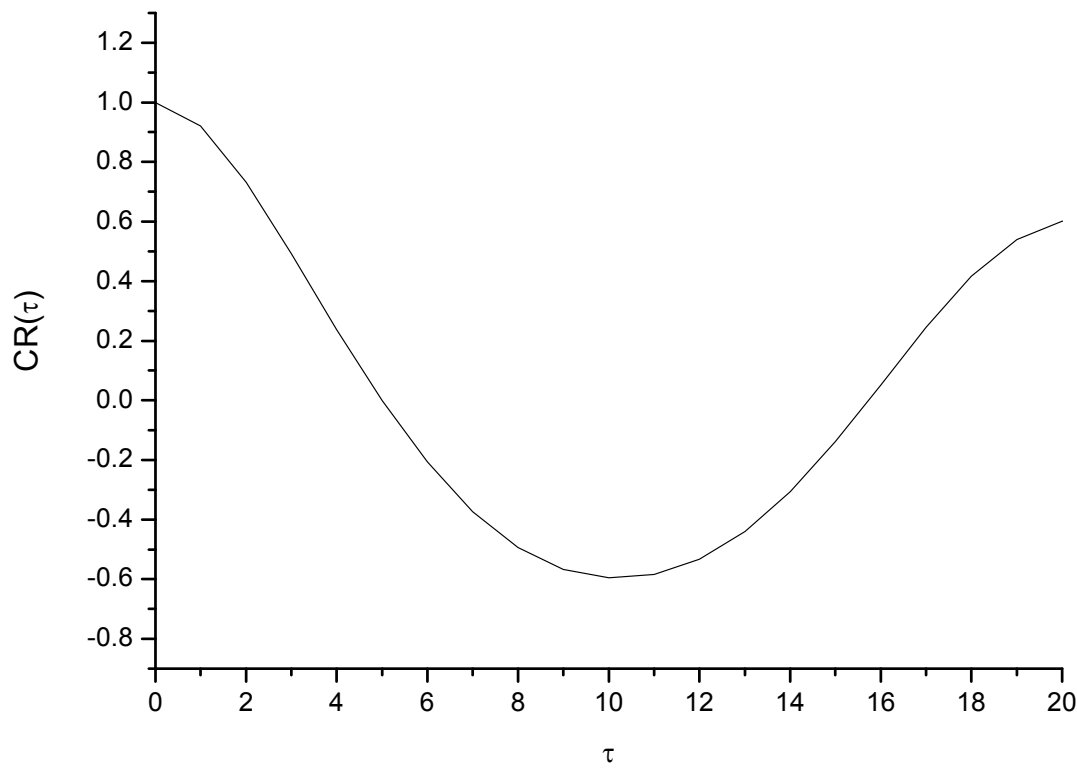

Fig. 4. Correlation function $C R(\tau)$ versus delay time $\tau$

It has been proven (Grassberger \& Procaccia, 1983) that if the attractor is a strange one, the correlation integral will be proportional to $r v$, where $v$ is a measure of the attractor's dimension called correlation dimension. By definition, the correlation integral $C(r)$ is the limit of correlation sum of equation (1) and is numerically calculated as a function of $r$, from equation (1), for embedding dimensions $m=1, \ldots, 10$. Figure 5 , depicts the relation between the logarithms of correlation integral $C(r)$ and $r$ for different embedding dimensions $m$. As seen in Figure 6, the slopes $v$ of the lower linear parts of these $\log$ - $\log$ curves provide all necessary information for characterizing the attractor. Then, in Figure 7 , the corresponding average slopes $v$ are given as functions of the embedding dimension $m$, indicating that for high values of $m, v$ tends to saturate at the non integer value of $v=2.23$. For this value, the minimum embedding dimension can be 3 (Kanz and Schreiber, 1997) and thus the minimum embedding dimension of the attractor for one to one embedding will be equal to 3 . 


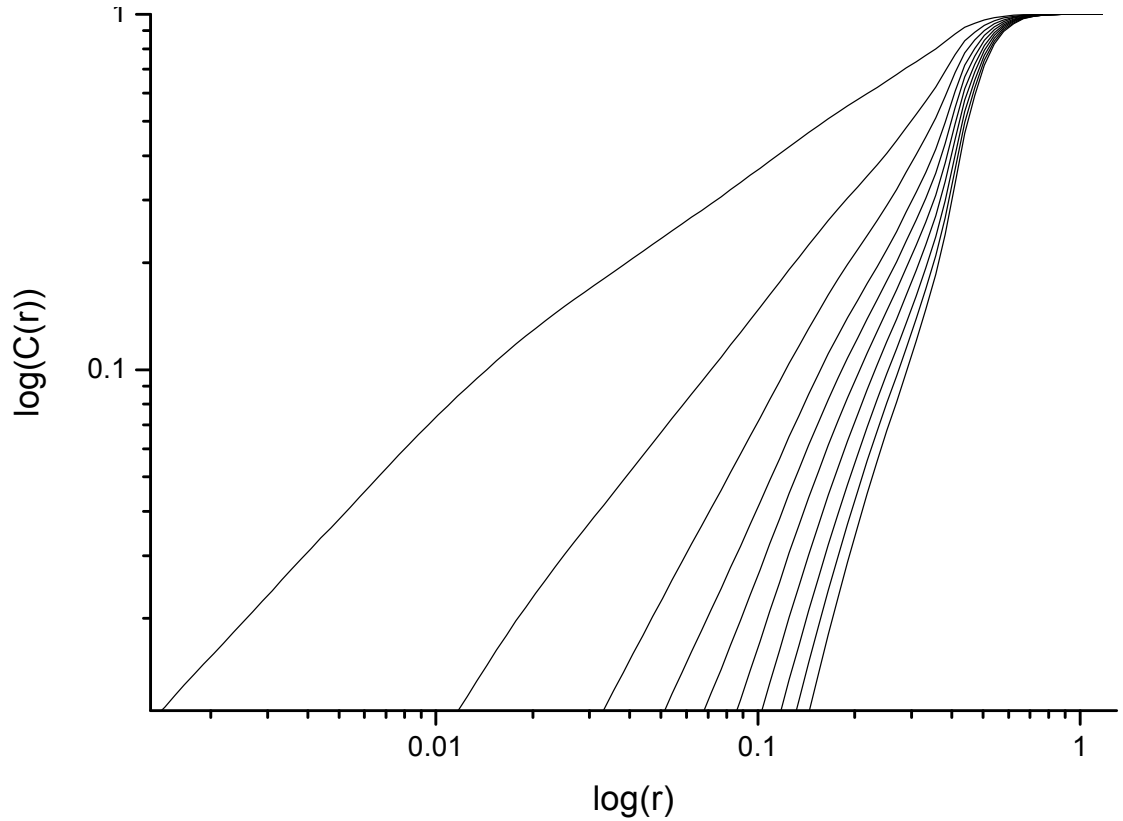

Fig. 5. Relation between $\log (C(r))$ and $\log (r)$ for different embedding dimensions $m$

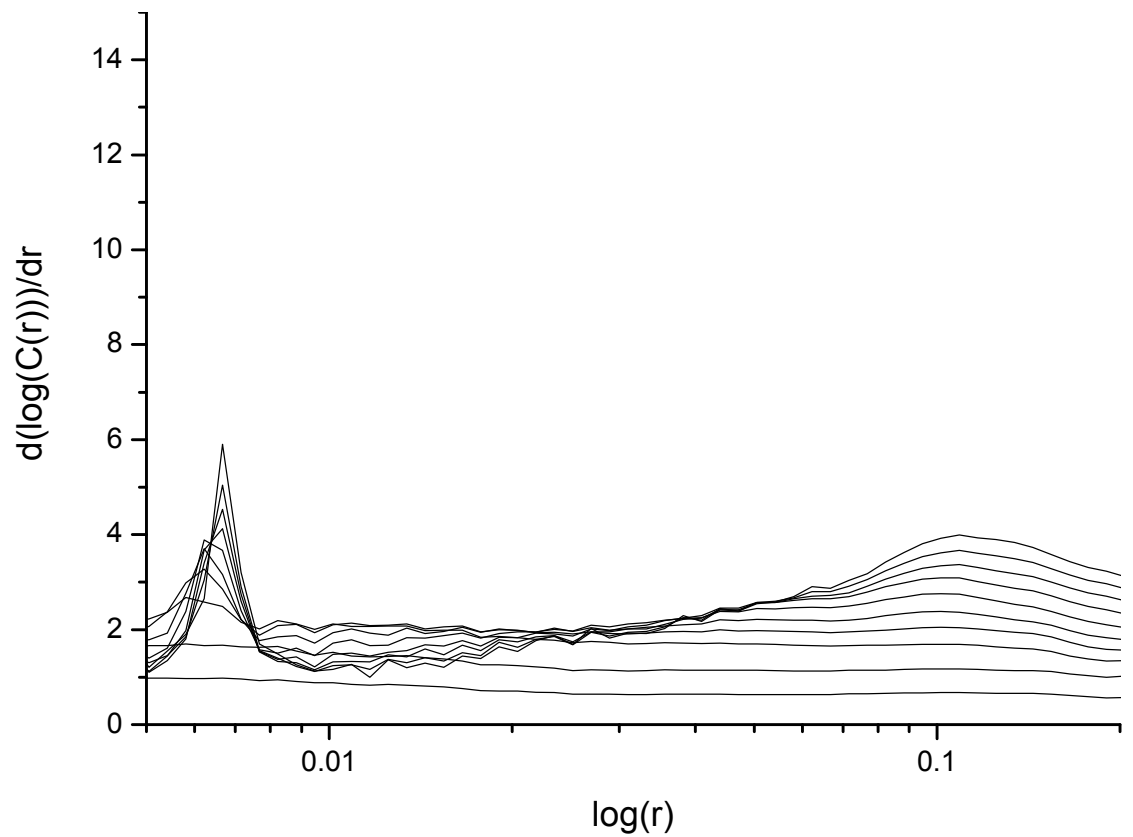

Fig. 6. The corresponding slopes and scaling region of Figure 5 


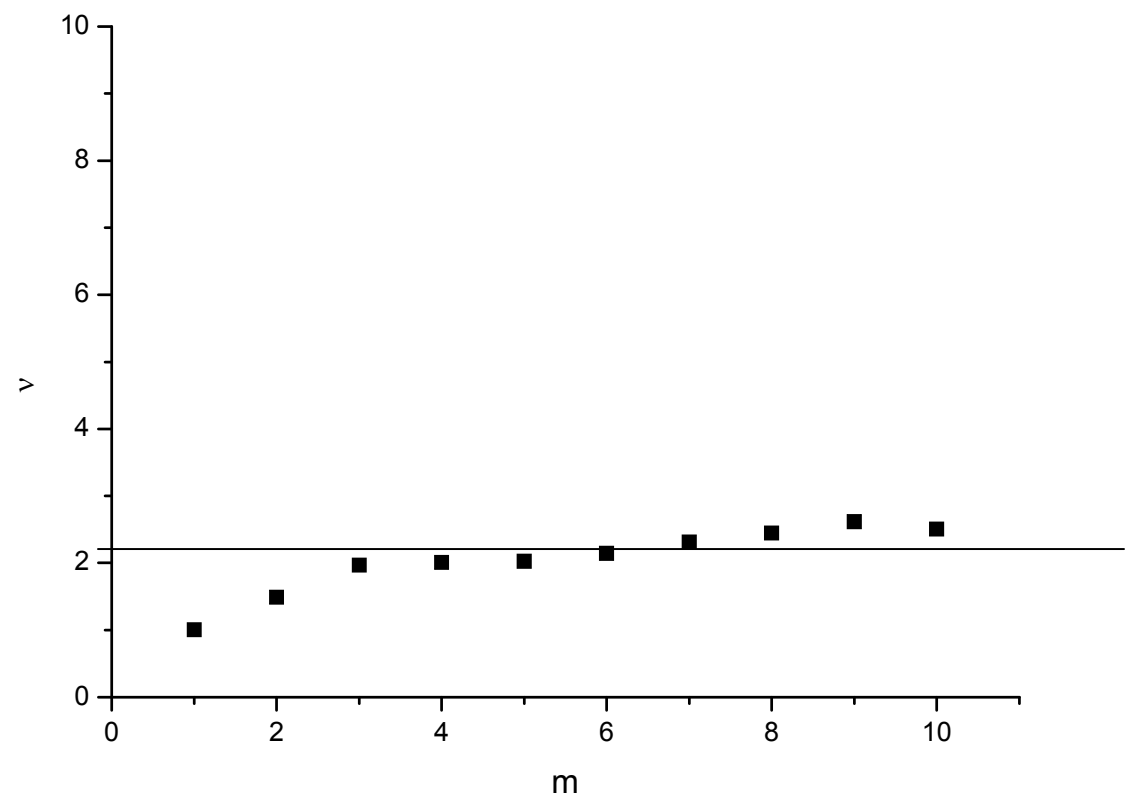

Fig. 7. Correlation dimension $v$ versus embedding dimension $m$

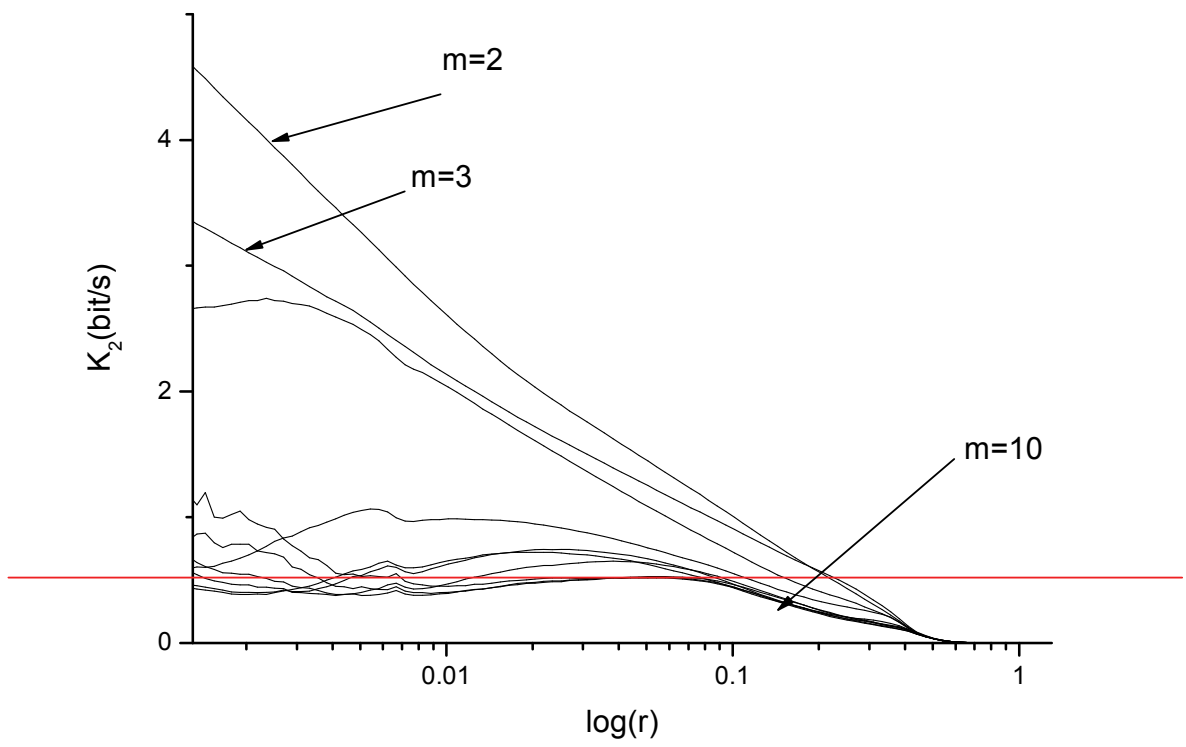

Fig. 8. Kolmogorov entropy versus $\log (r)$ for embedding dimensions $m=2, \ldots 10$ 
Following the above, in order to get accurate measurements of the strength of the chaos present in the oscillations of the simulated output signal, we introduce the Kolmogorov entropy. According to Kanz and Schreiber (Kanz \& Schreiber, 1997), the method followed so far, also leads to an estimation of the Kolmogorov entropy, i.e. the correlation integral $C(r)$ scales with the embedding dimension $m$, since:

$$
C(r) \sim e^{-m \tau_{d} K_{2}}
$$

where $K_{2}$ represents a lower bound to the Kolomogorov entropy. In figure 8 is shown the relation between $K_{2}$ and the logarithm of $r$ for different embedding dimensions $m$. It is clear from figure 8 that around $K_{2}=0.52 \mathrm{bit} / \mathrm{s}$ the trajectories appear a "plateau", a red line marks the region, which indicates that there is a steady loose of information at a constant rate given by $K_{2}$.

\section{Single chaotic optocoupling device}

\subsection{Description of the circuit}

There is a growing interest for non-autonomous chaotic signal generation circuits. Such a circuit may be externally driven and it can typically consist of only one active and a few passive components. Here, we consider a particularly simple circuit based on a single optocoupling device. Its complete layout is shown in figure 9 and it consists of a 4 N25 optocoupler, in a typical common emitter configuration, along with an emitter degeneration resistor $R_{2}=3.3 \mathrm{~K} \Omega$, a collector resistor $R=33 \Omega$, and a DC power supply $V_{S S}=12 \mathrm{~V}$. The circuit is driven by an input sinusoidal voltage $v_{I N}(t)$ with amplitude $V_{i n}=13 \mathrm{~V}$ and frequency $f=800$ $\mathrm{Hz}$, which is applied through an inductor $L=1 \mathrm{mH}$ connected in series to the driver LED and a resistor $R_{1}$, the value of which, as we will see, plays a crucial role in the circuit's operation and the generation of chaotic voltage time series across $R_{1}$ and $R_{2}$. In this respect, we use the MultiSim circuit simulation software in order to examine the complete circuit operation by monitoring the voltages $v_{D}(t)$ and $v_{E}(t)$ across $R_{1}$ and $R_{2}$, respectively.

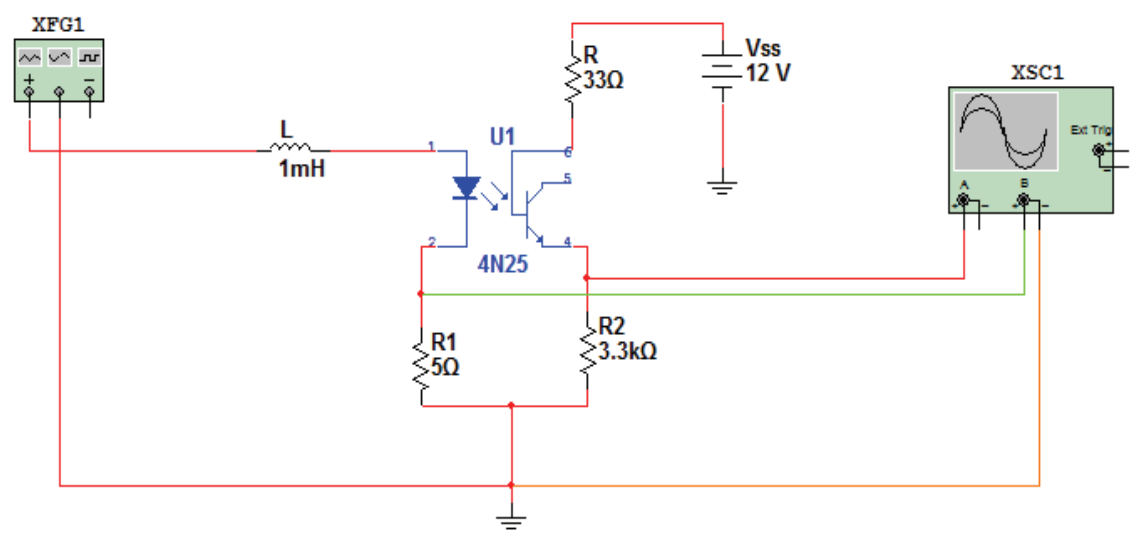

Fig. 9. The optocoupling circuit and its MultiSim simulation environment 
Here, we must note that the considered circuit layout resembles the resistor-inductor-diode (RLD) and the resistor-inductor-transistor (RLT) circuits, whose chaotic operation details have been presented and discussed in (Hanias \& Tombras, 2009). More specifically, following the conclusions derived in (Hanias \& Tombras, 2009), we use various values for $R_{1}$ in order to achieve chaotic operation in both, if it is possible, the input LED loop and the emitter output loop, i.e. across $R_{1}$ and $R_{2}$, respectively. Then, as mentioned above, the simulated circuit operation is monitored by checking the voltages $v_{D}(t)$, across $R_{1}$, and $v_{E}(t)$, across $R_{2}$, since both of these voltage signals depend on the chosen value of the LED resistor $R_{D}$.

\subsection{Simulation results}

For the considered circuit operation simulation, we use $v_{I N}(t)=V_{\text {in }} \sin (2 \pi f t)$ with $V_{\text {in }}=13$ Volts and $f=800 \mathrm{~Hz}$. It is known that, chaotic operation may be generated under various operation conditions and parameters' values. In this chapter, we choose to keep $V_{\text {in }}$ and $f$ constant, while varying the value of $R_{1}$. Our goal is to first examine whether chaos can be achieve for a specific $R_{1}$ value and then to examine whether variation of that value of $R_{1}$ may strengthen, weaken, or even destroy the achieved chaos, by returning the circuit to its typically expected operation.

Under these conditions and after some try-and-error selections for the value of $R_{1}$, we conclude that if $R_{1}$ is set equal to $5 \Omega$ then the circuit will exhibit a fully chaotic operation, meaning that both voltage signals $v_{D}(t)$ across $R_{1}$, and $v_{E}(t)$ across $R_{2}$, are chaotic. This is readily shown in figure 10. Then, using this as starting point, we see that an increase of $R_{1}$ weakens the already obtained chaotic signals and this continues up to a value of $R_{1}=500 \Omega$, for which a weak chaotic signal can still be seen across $R_{1}$, but not across $R_{2}$. This is shown in figure 11. Finally, further increase of $R_{1}$ leads to the total destruction of the remaining chaotic signal across $R_{1}$. This is shown in figure 12 where, for $R_{1}=1 \mathrm{~K} \Omega$, both voltage signals $v_{D}(t)$ and $v_{E}(t)$ are not chaotic.

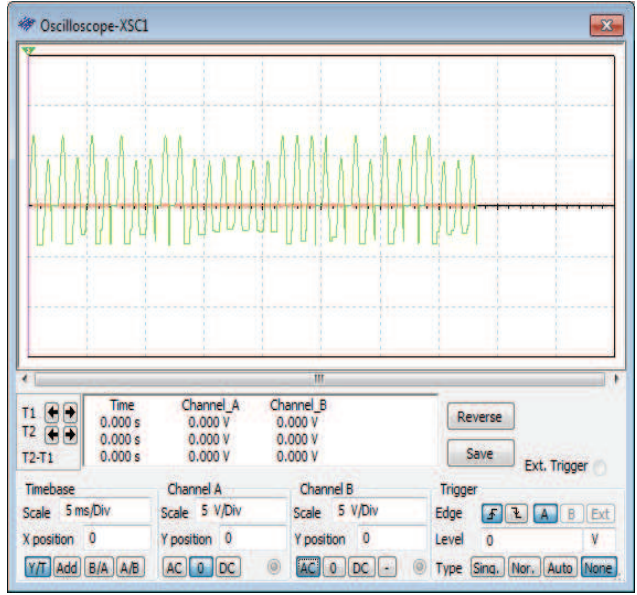

(a)

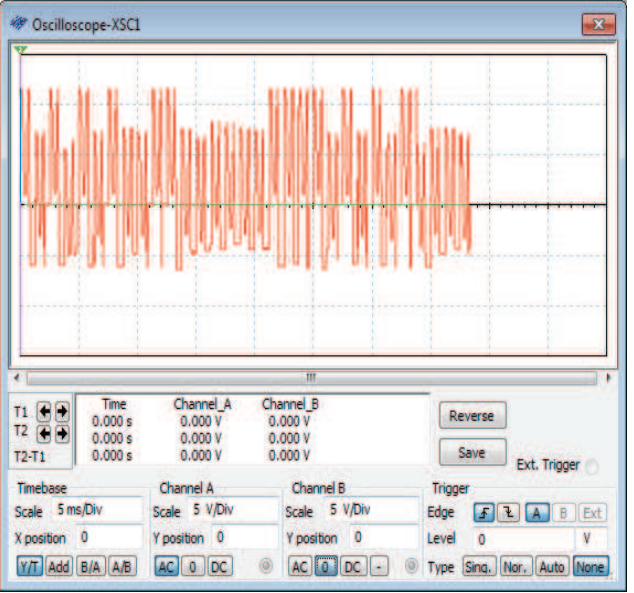

(b)

Fig. 10. For $R_{1}=5 \Omega$ : both voltage signals, (a) $v_{D}(t)$ across $R_{1}$ and (b) $v_{E}(t)$ across $R_{2}$, are chaotic 


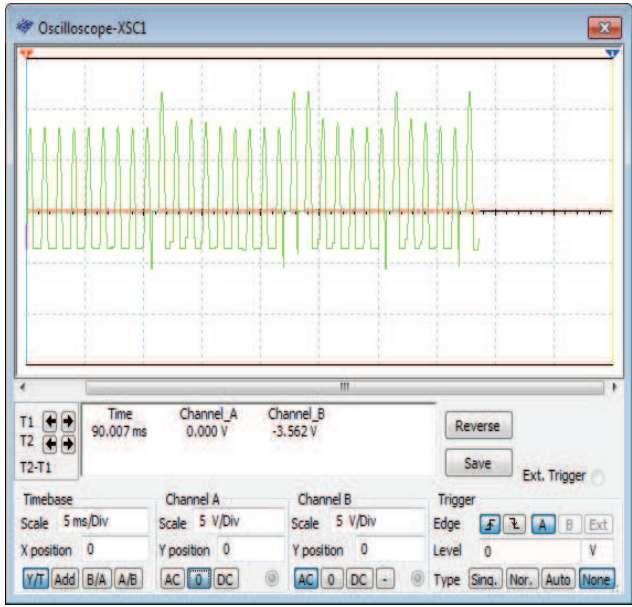

(a)

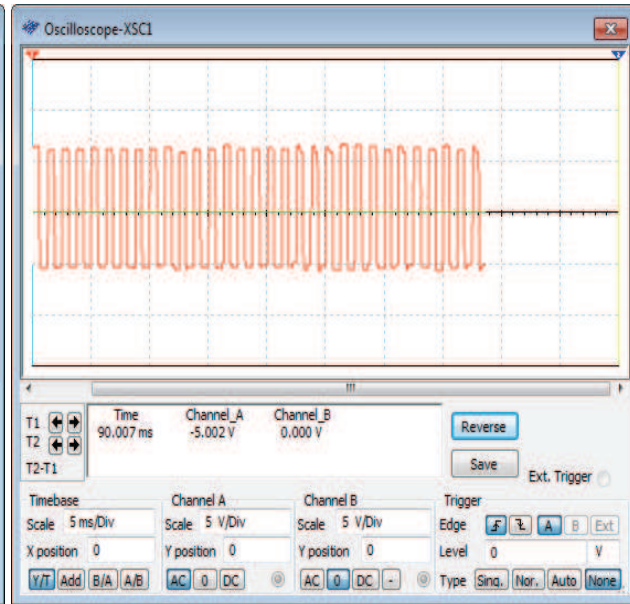

(b)

Fig. 11. For $R_{1}=500 \Omega$, (a) $v_{D}(t)$ is a weak chaotic signal across $R_{1}$ and (b) $v_{E}(t)$, across $R_{2}$, is non chaotic

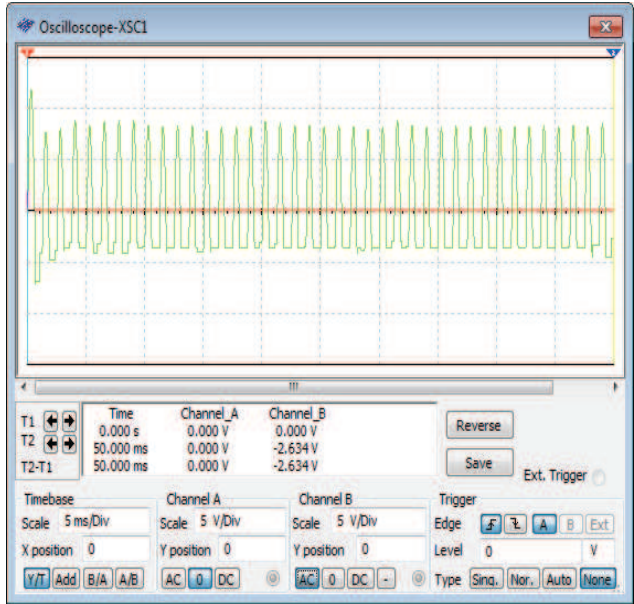

(a)

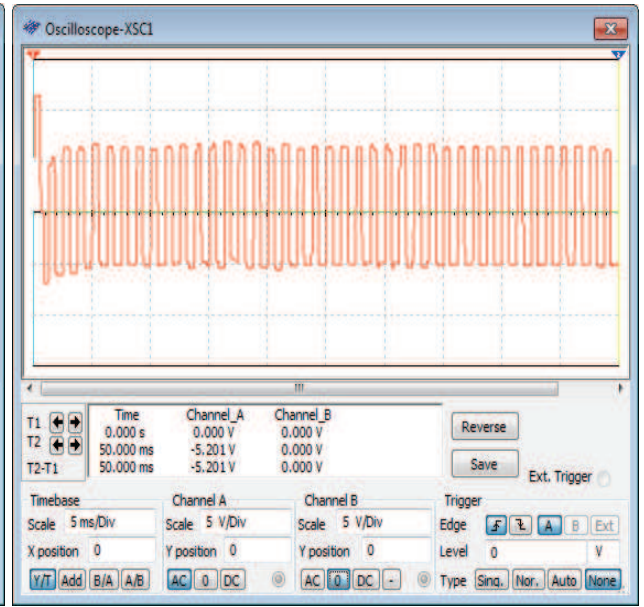

(b)

Fig. 12 . For $R_{1}=1 \mathrm{~K} \Omega$, both voltage signals (a) $v_{D}(t)$ across $R_{1}$ and (b) $v_{E}(t)$ across $R_{2}$, are non chaotic

\subsection{Nonlinear analysis}

In this section, we proceed to the analysis of the obtained chaotic signals time series when $R_{1}=5 \Omega$. Using our data, with value of $R_{1}=5 \Omega$ we construct a vector $\vec{X}_{i}, i=1$..N, where $N=5000$ data values in the $\mathrm{m}$ dimensional phase space given in the following form: 


$$
\vec{X}_{i}=\left\{v_{t}, v_{i-\tau}, v_{i-2 \tau}, \ldots v_{i-(m-1) \tau}\right\}
$$

This vector, represents a point of the $m$ dimensional phase space in which the attractor is embedded each time, where $\tau$ is the time delay $\tau=\mathrm{i} \Delta t$ and $\Delta t=0.1 \mu$ s is the sample rate. The element $v_{i}$ represents a value of the examined scalar time series in time, i.e. here a voltage value $v *$, across $R_{1}$ or $R_{2}$, corresponds to the i-th component of the time series. The use of this method reduces phase space reconstruction to a problem of proper determination of suitable values of $m$ and $\tau$. The choice of these values is not always simple, especially when there is no additional information about the original system and the only source of data is a simple sequence of scalar values as acquired from that system.

The dimension, where a time delay reconstruction of the phase space provides for the necessary number of coordinates to unfold the dynamics from overlaps on itself caused by projection is called embedding dimension $m$. Using the average mutual information, we can then obtain $\tau$ as being less associated with a linear point of view, and thus, more suitable for dealing with nonlinear problems. The average mutual information $I(\tau)$, expresses the amount of information (in bits), which may be extracted from the value in time $v_{i}$ about the value in time $v_{i+\tau}$. As optimum $\tau$, suitable for the phase space reconstruction, the position of the first minimum of $\mathrm{I}(\tau)$ is usually used. In this case $\tau=77$ time steps for chaotic signal across emitter resistor $R_{2}$ and $\tau=38$ steps for chaotic signal across LED resistor $R_{1}$ as shown in figure 13.

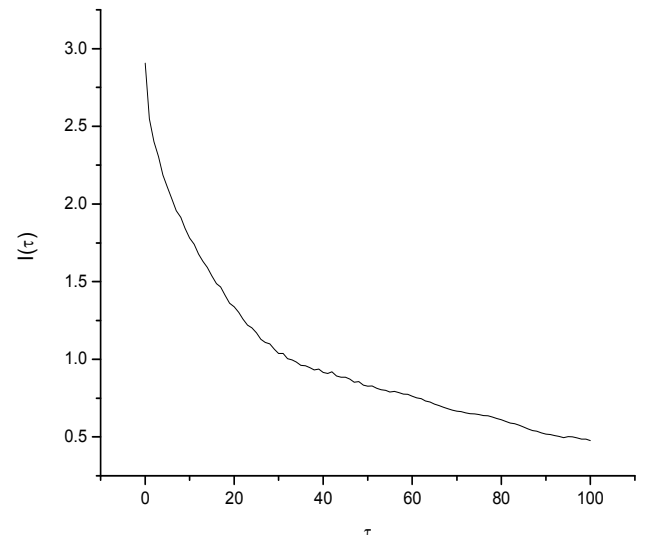

(a)

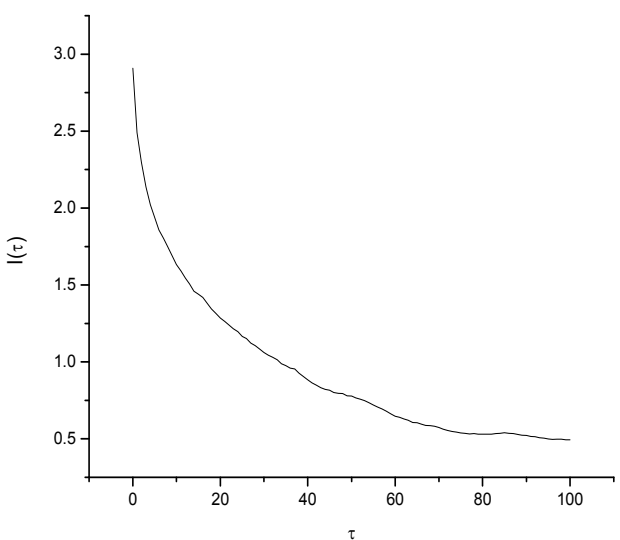

(b)

Fig. 13. Mutual information $I$ versus time delay $\tau$ for both chaotic signals across (a) $R_{1}=5 \Omega$ and (b) $R_{2}$

Next, we use the method of False Nearest Neighbors (FNN), [Hanias et al., 2010], in order to estimate the minimum embedding dimension. This method is based on the fact that when the embedding dimension is too small, the trajectory in the phase space will cross itself. Hence, if we are in position to detect these crossings, we may decide whether the used $m$ is large enough for correct reconstruction of the original phase space [i.e. when no intersections occur] or not. If, however, intersections are present for a given $m$, then the embedding dimension must be considered too small and it must be increased by one at least. Then, again, we test the eventual presence of self-crossings. 
The practical realization of the described method is based on testing the neighboring points in the $m$-dimensional phase space. Typically, we take a certain amount of points in the phase space and find the nearest neighbor to each of them. Then we compute distances for all these pairs and also their distances in $(m+1)$-dimensional phase space. The rate of these distances is given by

$$
P=\frac{\left\|\vec{X}_{i}(m+1)-\vec{X}_{n(i)}(m+1)\right\|}{\left\|\vec{X}_{i}(m)-\vec{X}_{n(i)}(m)\right\|}
$$

where $\vec{X}_{i}(m)$ represents the reconstructed vector as described in (1), belonging to the i-th point in the $m$-dimensional phase space and index $n(i)$ denotes the nearest neighbor to the $i$ th point. If $P$ is greater than some value $P_{\max }$, we call this pair of points false nearest neighbors (i.e. neighbors, which arise from trajectory self-intersection and not from the closeness in the original phase space. The dimension $m$ will then be found when the false nearest neighbors percentage falls below some limit, typically set to 1\%, [Kennel et al.,1992]. Thus, by choosing $P_{\max }=10$, we finally calculate the quantity $m$. The so obtained results are shown in figure 14 indicating that the application of the FNN method yields a minimum embedding equal to value 5 for both chaotic signals.

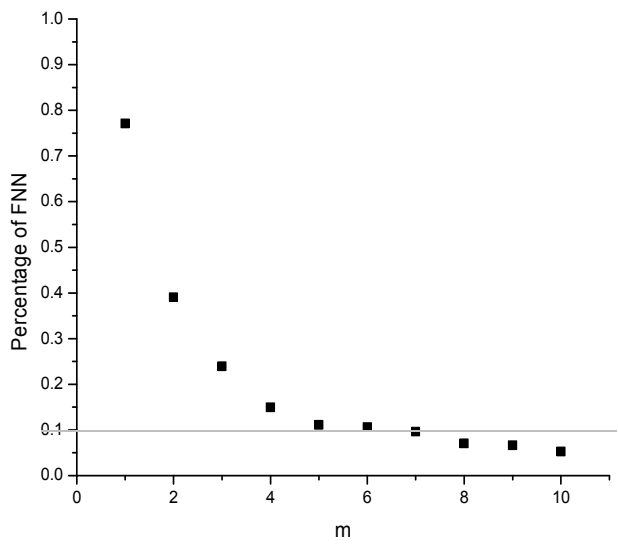

(a)

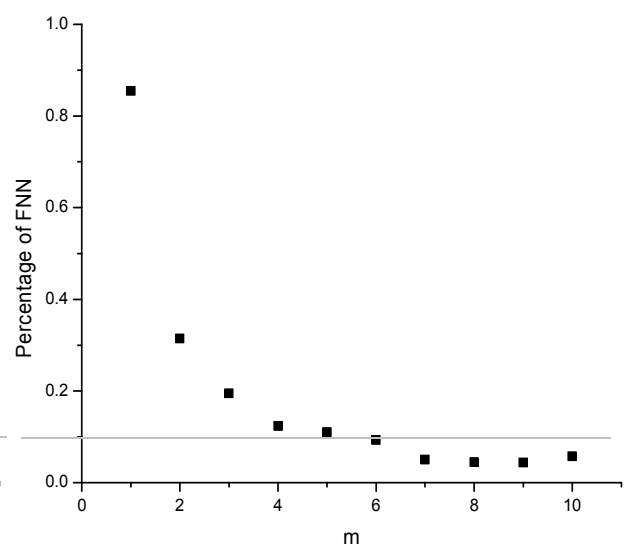

(b)

Fig. 14. False nearest neighbor ratio as a function of the embedding dimension. The false nearest neighbors become negligible after $m=5$ for the chaotic signals (a) across LED resistor $R_{1}=5 \Omega$ and (b) across emitter resistor $R_{2}$

\section{Optoelectronic simulation of the Duffing-Holmes equation}

\subsection{Description of the circuit}

It is well known that the extremely simple analogue electrical circuit dedicated for simulation the Duffing-Holmes (DH) equation (Holmes, 1979). Our approach is to modify the circuit E (Tamaseviciute et al., 2008), by adding a LED component and finding the various operation conditions and parameters' values that enhanced or not the chaotic 
operation of LED component. The suggested circuit is shown in figure 15. It is an externally driven damped RLC oscillator with all elements linear. The nonlinearity is involved by the positive feedback loop consisting of the resistor $R_{3}$ and two diodes $D_{1}$ and $D_{2}$. The operational amplifier OA plays the role of both, the buffer for the external sinusoidal force and the amplifying stage for the positive nonlinear feedback. The electrical circuit resembles the Young-Silva oscillator (Lai et al., 2005), but is essentially simpler. It includes a single operational amplifier, two diodes, and four resistors only, in contrast to the Young-Silva circuit containing four operational amplifiers, four diodes, and nine resistors

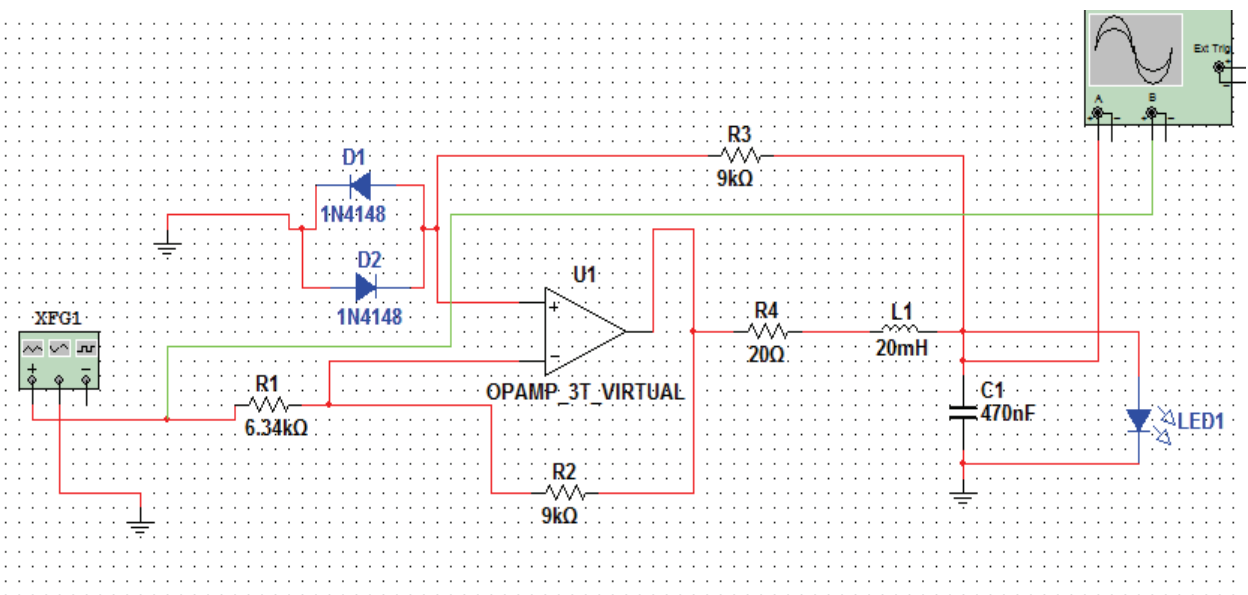

Fig. 15. The considered optoelectronic Duffing-Holmes circuit and its MultiSim simulation environment

The circuit in figure 15 has been simulated using the "Electronics Workbench Professional" package which is based on the SPICE software. The following circuit element values have been used: $L=20 \mathrm{mH}, C=470 \mathrm{nF}, R_{1}=6.34 \mathrm{~K} \Omega, R_{2}=9 \mathrm{~K} \Omega, R_{3}=9 \mathrm{~K} \Omega, R_{4}=20 \Omega$. The operational amplifier OA is virtual one but the circuit works the same way with a LM741 type chip, the diodes $D_{1}$ and $D_{2}$ are general-purpose 1 N4148 or similar type silicon devices. Simulation results are shown in figure 16 and figures 17-18. The circuit is driven by an input sinusoidal voltage with amplitude $V_{\text {in }}=200 \mathrm{mV}$ and frequency $f=1.5 \mathrm{kHz}$

\subsection{Simulation results}

It is known that, chaotic operation may be generated under various operation conditions and parameters' values. In this work, we keep $V_{\text {in }}$ and $f$ constant, while varying the value of $R_{1}$. Our aim is to first examine whether chaos can be achieve for a specific $R_{1}$ value and then to examine whether variation of that value $R_{1}$ may strengthen, weaken, or even destroy the achieved chaos, by returning the circuit to its typically expected operation. Under these conditions and after some try-and-error selections for the value of $R_{1}$, we conclude that if $R_{1}$ is set equal to $5.76 \mathrm{~K} \Omega$ then the circuit will exhibit a periodic operation. This is readily shown in figure 16. Then, using this as a starting point, we see that an increase of $R_{1}$ the circuit will exhibit a fully chaotic operation. This is shown in figure 17 for $R=6 \mathrm{~K} \Omega$ and in figure 18 for $R=6.81 \mathrm{~K} \Omega$. 


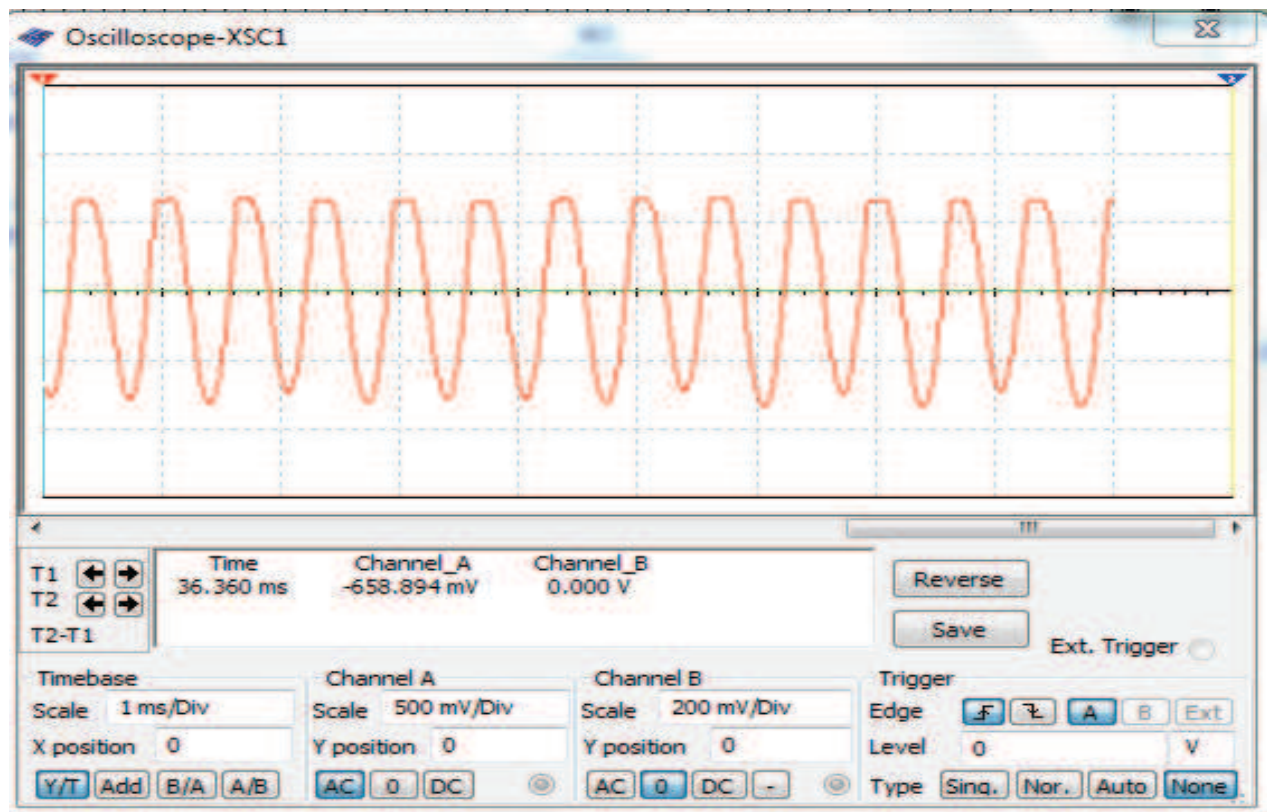

Fig. 16. For $R_{1}=5.76 \mathrm{~K} \Omega$ the voltage signal and LED response are periodic

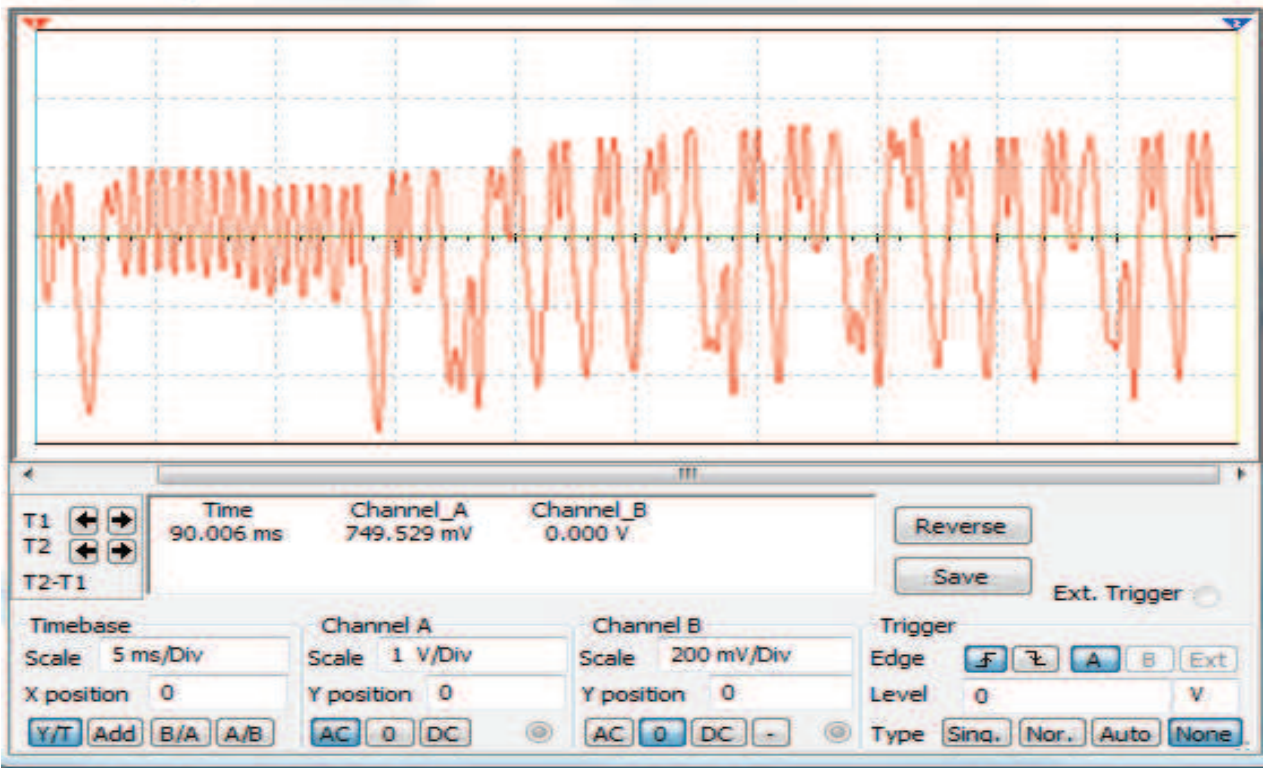

Fig. 17. For $R_{1}=6 K \Omega$, the voltage signal and LED response are chaotic 


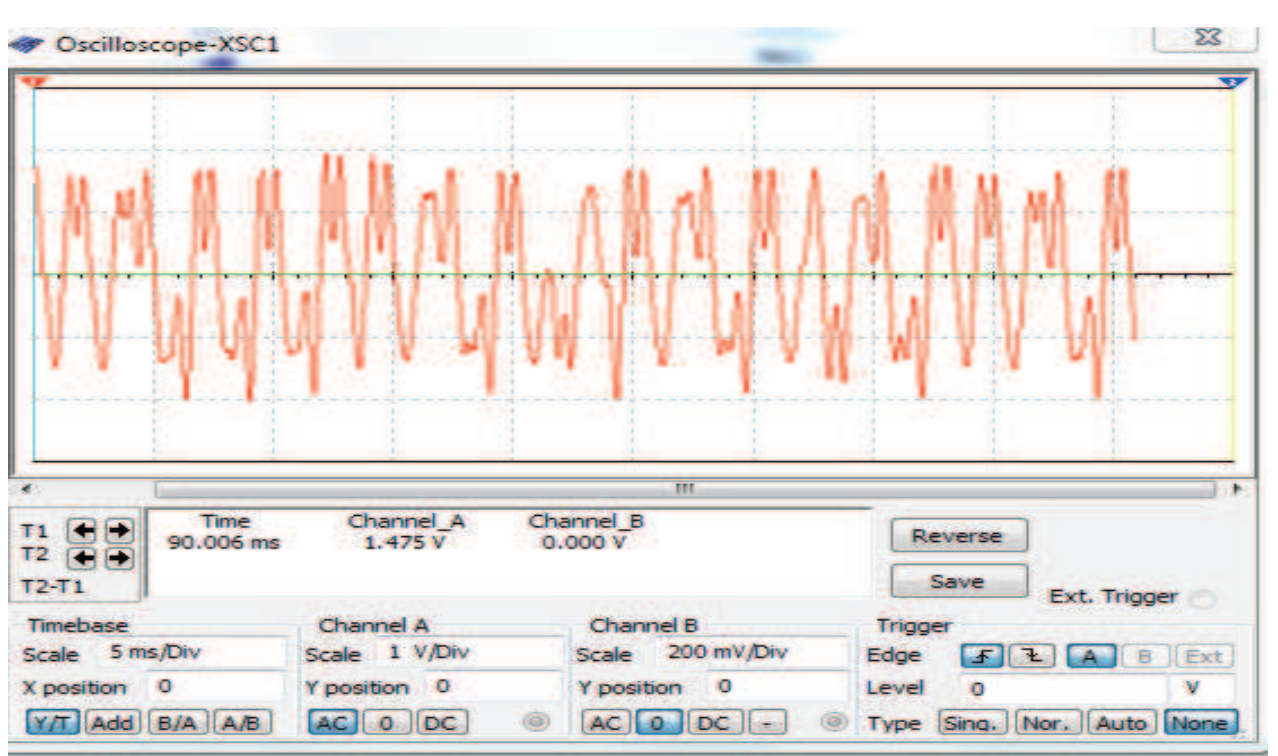

Fig. 18. For $R_{1}=6.81 \mathrm{~K} \Omega$ the voltage signal and LED response are chaotic

\subsection{Nonlinear analysis}

We apply the analysis as we did in section 2.2. For $R_{1}=6.8 \mathrm{k} \Omega$ we examine the signal across the LED while the LED is in operation mode The signal is shown in figure 18 . We record $N=13454$ points with sample rate $\Delta t=6.66 \mu \mathrm{s}$. As shown in figure 19 , the mutual information function exhibits a local minimum at $\tau=26$ time steps and, thus, we shall consider $\tau=26$ as the optimum delay time.

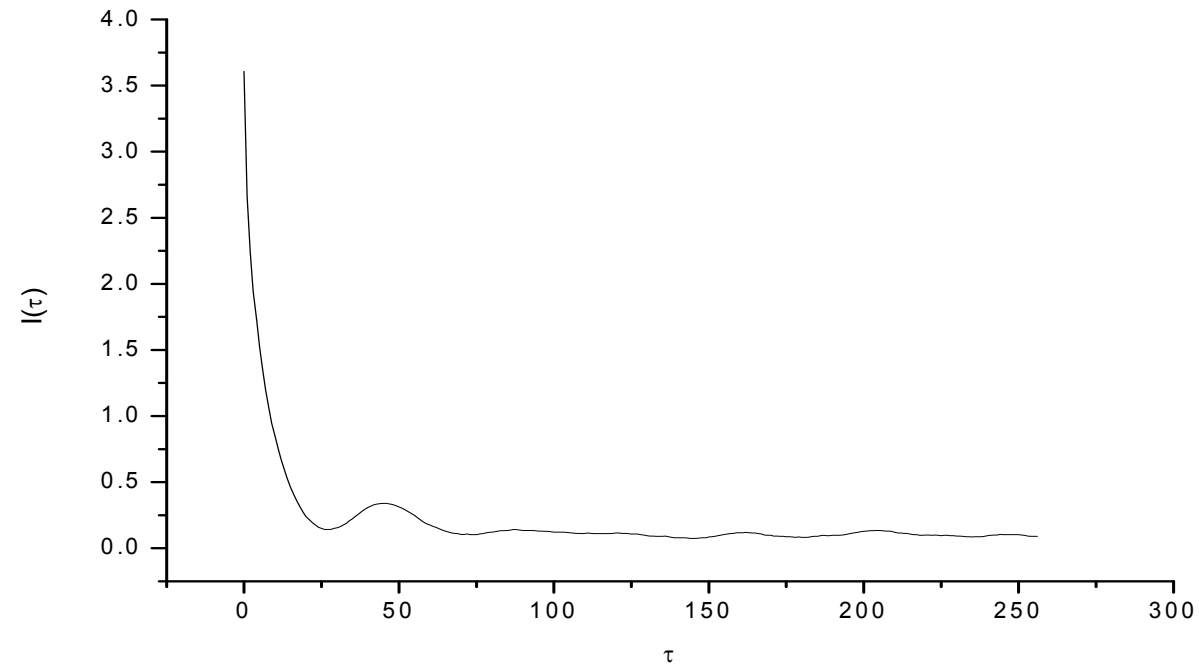

Fig. 19. Average Mutual Information $I(\tau)$ versus time delay $\tau$ 


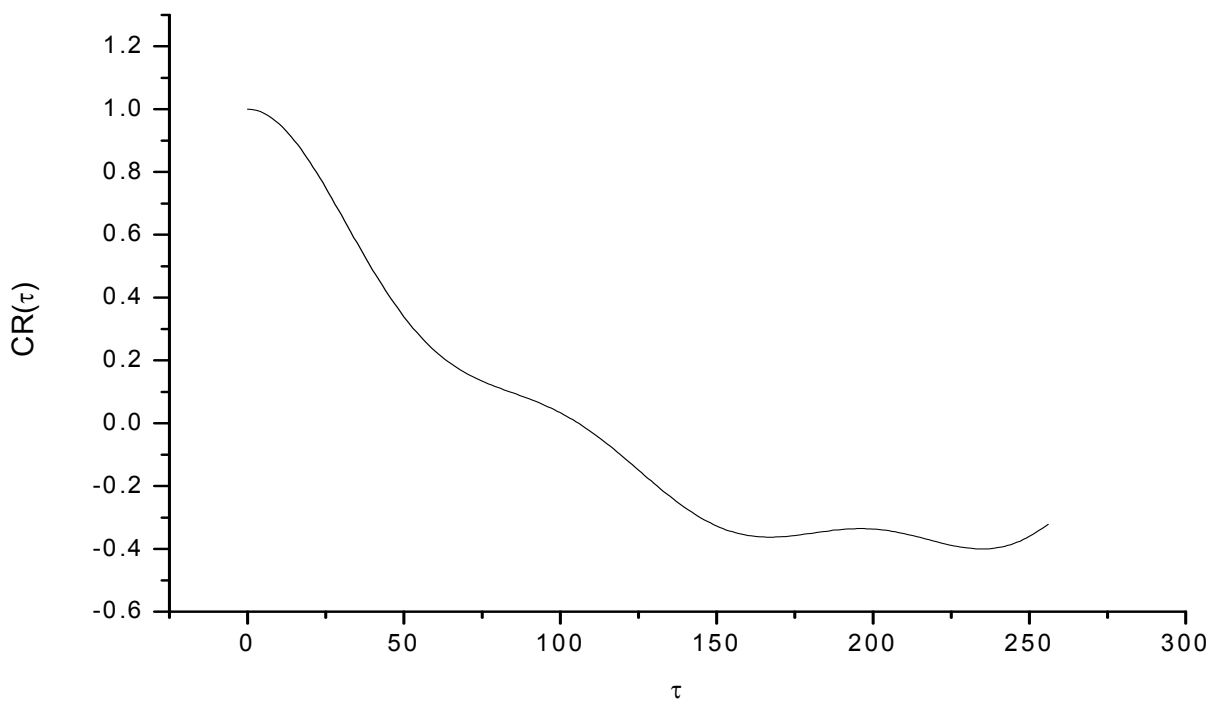

Fig. 20. Correlation function $C R(\tau)$ versus time delay $\tau$

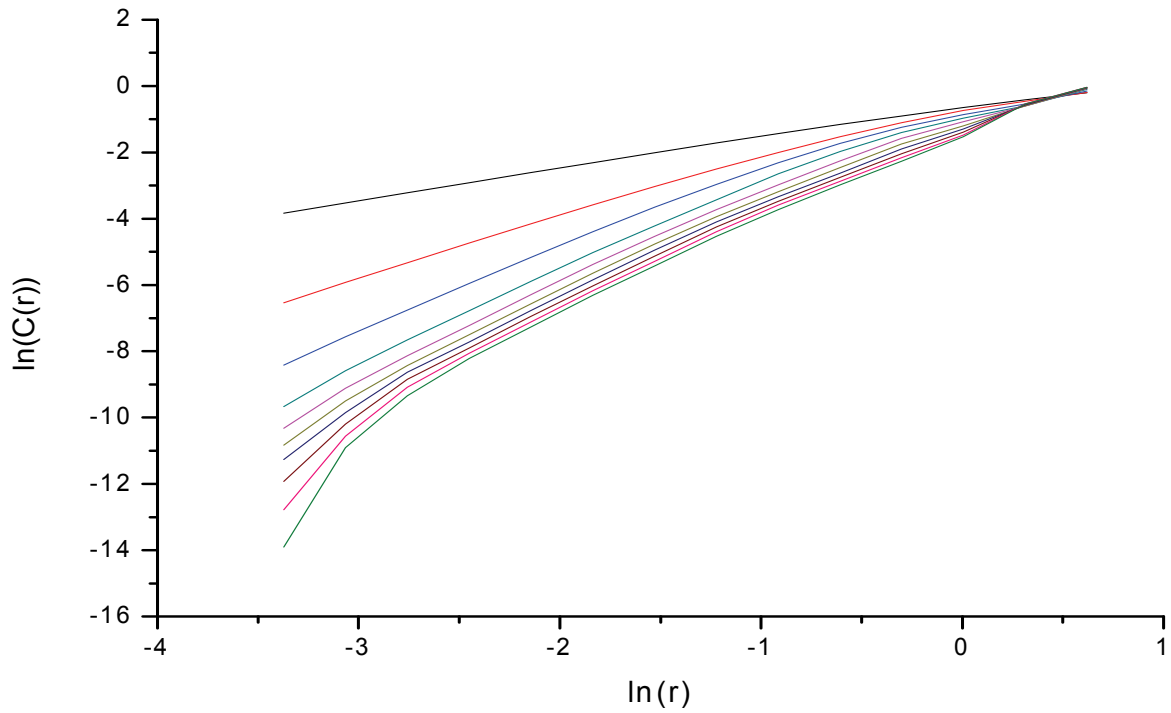

Fig. 21. Relation between $\ln (C(r))$ and $\ln (r)$ for different embedding dimensions $m$

Next we calculate the autocorrelation function as we did in section 2.2 As shown in figure 20 , the first zero crossing is at $\tau=107$, then the correlation length is equal to 107 and thus, $W=107$ time lags. Hence, we can use these values for phase space reconstruction. 


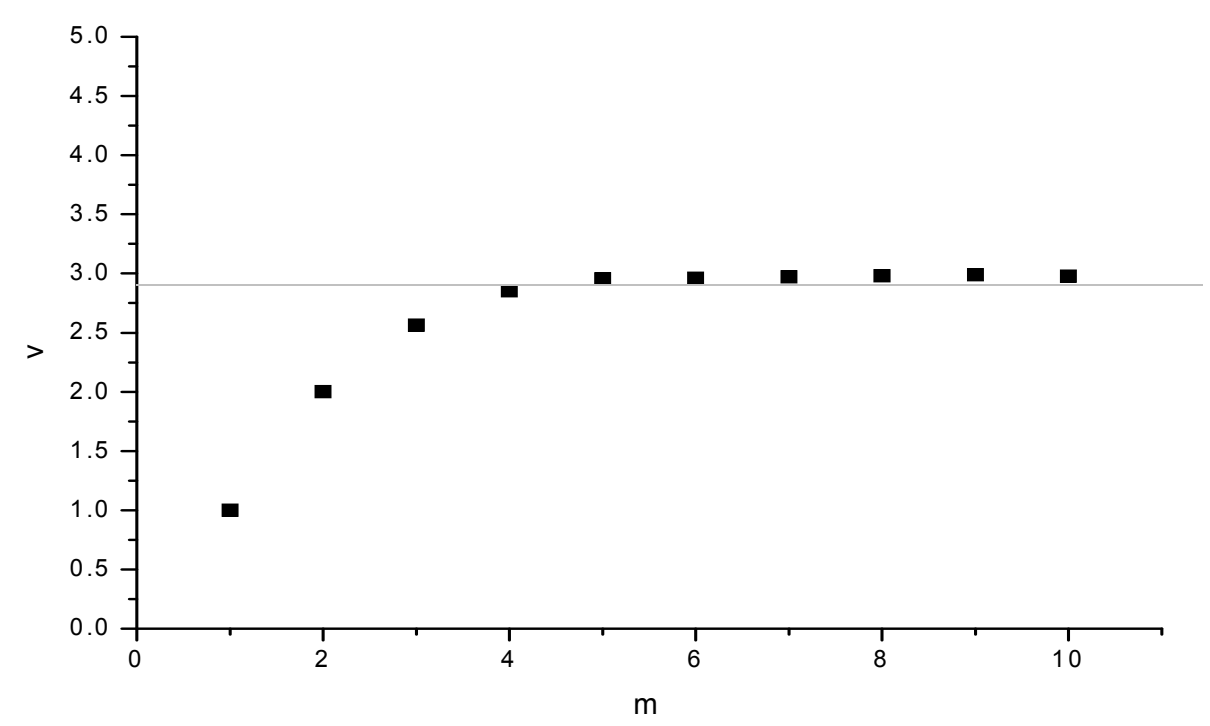

Fig. 22. Correlation dimension $v$ versus embedding dimension $m$

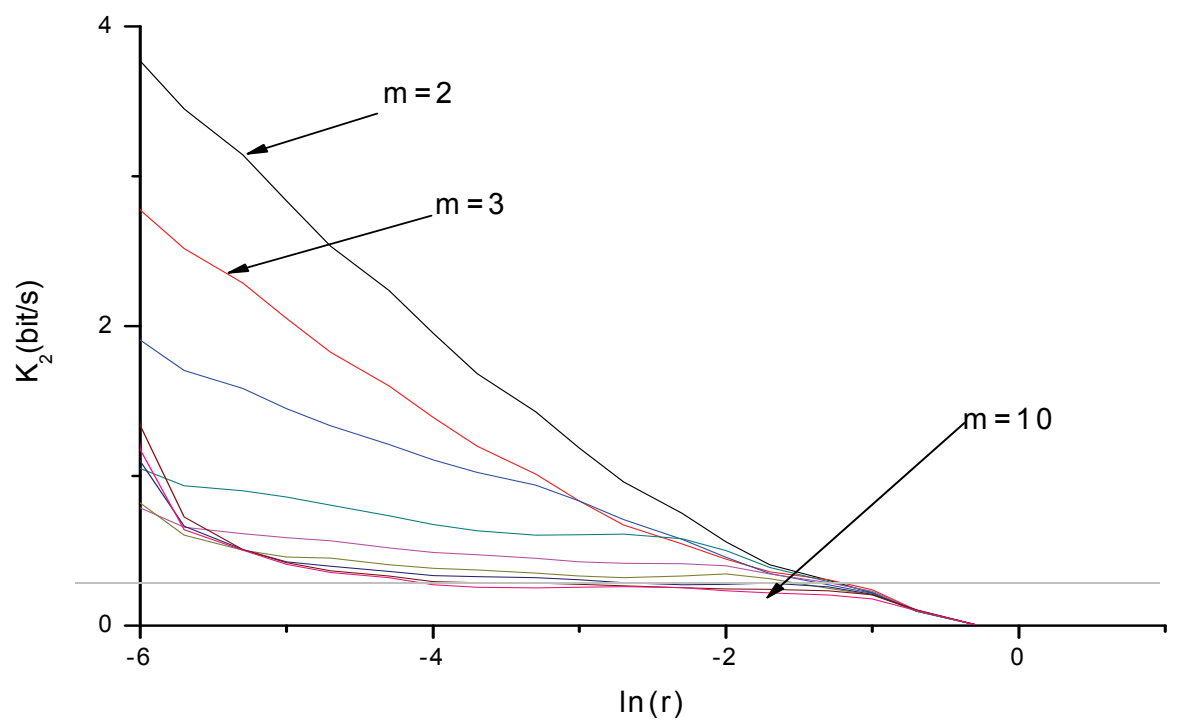

Fig. 23. Kolmogorov entropy versus $\ln (r)$ for embedding dimensions $m=2, \ldots 10$

Figure 21 depicts the relation between the natural logarithms of correlation integral $C(r)$ and $r$ for different embedding dimensions $m$. In figure 22, the corresponding average slopes $v$ are given as a function of the embedding dimension $m$ indicating that for high values of $m, v$ tends to saturate at the non integer value of $v=2.9$. For this value of $v$, the minimum embedding dimension can be $m_{\min }=3$ and thus, the minimum embedding dimension of the attractor for one to one embedding will be equal to 3 . 
The Kolmogorov entropy is calculates as in section 2.2. Figure 23 shows the relation between $K_{2}$ and the logarithm of $r$ for different embedding dimensions $m$, while the plateau, indicates that $K_{2}=0.281 \mathrm{bit} / \mathrm{s}$, meaning that there is a steady loose of information at a constant rate given by $K_{2}$.

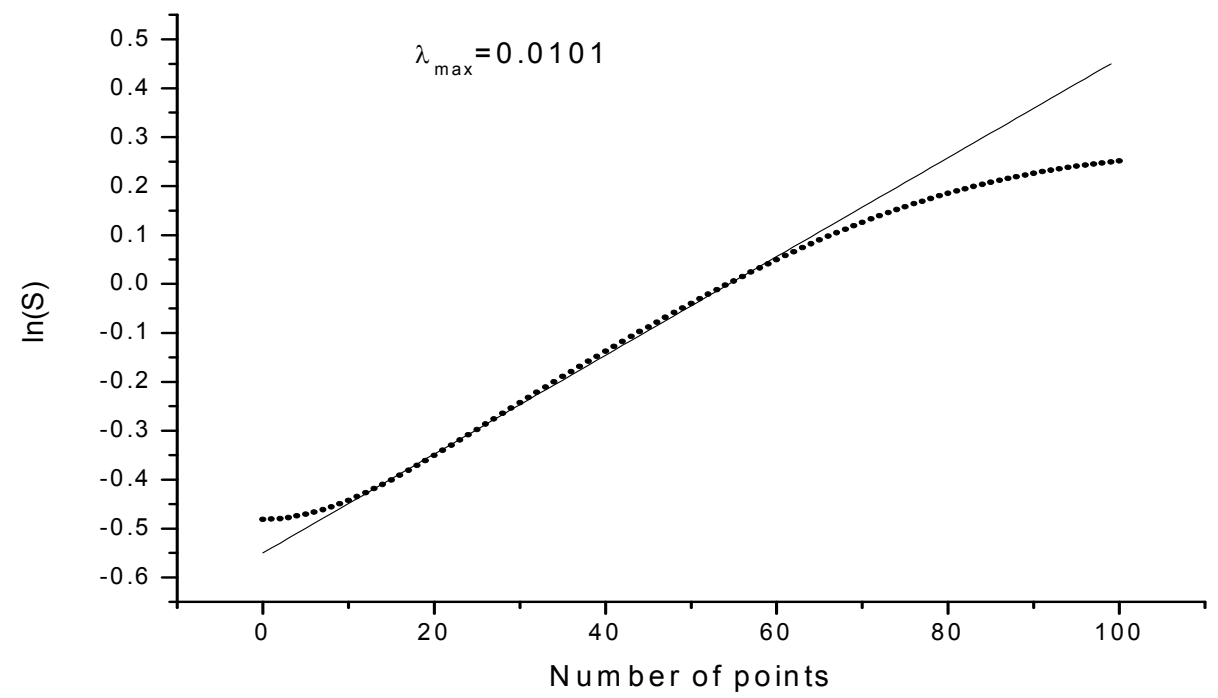

Fig. 24. Estimation of the largest Lyapunov exponent after the Rosenstein method. The portion of the curve used for the least-squares line fits the starting point up to the saturation point. The straight line, represents the resulting least-squares line fit with slope of 0.00101 for $m=3$

In order to determine the strength of the observed chaotic behaviour, we calculate the largest Lyapunov exponent, which measures the divergence of nearby trajectories. As the system evolves, the sum of a series of attractor point values (in each dimension) will either converge or diverge. Lyapunov exponents measure this convergence/divergence rate in each dimension and a chaotic system will exhibit trajectory divergence in at least one dimension. Thus, a positive Lyapunov exponent is a strong indicator of chaos (Rosenstein et.al., 1993). The method used in calculating the largest Lyapunov exponent is based on averaging the local divergence rates or the local Lyapunov exponents. Rosenstein in (Rosenstein et.al., 1993) proposed a new method to calculate the largest Lyapunov exponent from an observed times series. Hence, after reconstructing the phase space using suitable values for $\tau$ and $m$, we can compute the logarithm of the average distance of a point $X_{n 0}$ in phase space with respect to all points $X_{n}$ in its $r$-neighbourhood. This is repeated for $N$ number of points along the orbit so as to calculate an average quantity $S$ known as the stretching factor and given by

$$
S=\frac{1}{N} \sum_{n 0=1}^{N} \ln \left[\frac{1}{\left|u_{X_{n 0}}\right|} \sum\left|X_{n 0}-X_{n}\right|\right]
$$


Where $u_{X_{n 0}}$ is the number of neighbours found around point $X_{n 0}$. In the case of chaotic dynamics, a plot of the stretching factor $S$ against the number of points $N$ will yield a curve with a linear increase at the beginning, followed by an almost flat region. The slope of the linear portion of the first part of this curve gives an estimate of the largest Lyapunov exponent. The value of $r$ is taken as the data interval is divided by 200 and again, in order to avoid temporal correlations we use the Theiler window of 107 . The magnitude $\ln (S)$ versus the number of points $N$ shows the expected linear increase and then after a flat region. This is clearly shown in figure 9, where the slope value that corresponds to the largest Lyapunov exponent is obtained after the least-squares line fit and is found to be equal to $\lambda_{\max }=0.0101$ for $m=3$. Moreover, the dependence of $\lambda_{\max }$ on the embedding dimension $\mathrm{m}$ is shown in figure 24 .

\section{Conclusion}

From the previous analysis, it is clear, that simple optoelectronic circuits can easily constructed and also be easily controlled via a control parameter as is the value of Resistor or the frequency of voltage source. For RL -LED circuits the result is that the scaling behaviour of the correlation integral and the saturation of correlation dimension $v$ with increasing embedding dimensions $m$ reflect low dimensionality. The strange attractor that governs the phenomenon has a correlation dimension $v=2.23$ stretching and folding in a 3 dimension phase space. Thus, the number of degrees of freedom of the whole domain structure is limited at 3 and these results in the low value of the correlation dimension. The LED exposes chaotic behaviour even if it works in its operation point. In this work, the obtained simulation results indicate that the proposed circuit can be used to generate chaotic signal, in a light emitting manner, useful in code and decode applications. The Optoelectronic simulation of the Duffing-Holmes Equation is governed by a strange attractor that stretching and folding in a 3 dimension phase space too.

The higher value of its correlation dimension 2.9 instead of 2.23 of RL -LED circuit shows that this circuit is better for secure transmission of information using chaos while the loss of information is $K_{2}=0.281 \mathrm{bit} / \mathrm{s}$ instead of $0.52 \mathrm{bit} / \mathrm{s}$ of RL -LED circuit.

For the optocoupler circuit the chaos is generated easily. It is clear that this simple externally triggered optoelectronic circuit can be used in order first to generate chaotic voltage signals and then to control the obtained chaotic signals by varying specific circuit parameters, for example, the value of a specific component. In the considered optoelectronic circuit, the crucial parameter is the input loop resistor $R_{1}$, the value of which leads to the generation of a chaotic voltage signal $v_{D}(t)$ across the resistor $R_{1}$. However, this chaotic voltage reflects a chaotic current through $R_{1}$ and thus, through the optocoupler's input driving LED. Hence, the so driven phototransistor will generate a chaotic voltage signal $v_{E}(t)$ across emitter resistor $R_{2}$, according to the strength of the driving chaotic light signal as controlled by the value of the input loop resistor $R_{1}$.

\section{References}

Argyris, A.; Syvridis, D.; Larger, L.; Annovazzi-Lodi, V.; Colet, P.; Fischer, I.; García-Ojalvo, J. \& Shore, K.A.;(2005). Chaos-based communications at high bit rates using commercial fibre-optic links, Nature, vol. 438, no. 7066, pp. 343-346. 
Argyris, A.; Grivas, E.; Hamacher, M.; Bogris, A. \& Syvridis, D. (2010). Chaos-on-a-chip secures data transmission in optical fiber links, OPTICS EXPRESS, Vol. 18, No. 5 ,pp 5188-5198.

Grassberger, P \& Procaccia, I. (1983). Characterization of Strange Attractors, Phys. Rev Lett., vol. 50, pp. 346-349.

Hanias, M.P. \& Anagnostopoulos, J.A.N. (1993). Negative-differential-resistance effects in the TlGaTe2 ternary semiconductor, Phys. Rev B., vol. 47, pp. $4261-4267$.

Hanias, M.P.; Magafas, L. \& Kalomoiros, J. (2008). Non- linear Analysis in IN RL-LED optoelectronic circuit, Optoelectronics and advanced materials - Rapid Communications, vol. 2, no. 2, pp. $126-129$.

Hanias, M.P. \& Tombras, G.S. (2009). Time series analysis in single transistor chaotic circuit, Chaos, Solitons and Fractals, vol. 40, pp.246-256.

Hanias, M.P. \& Tombras G.S. (2009). Time series crossprediction in single transistor chaotic circuit, Chaos, Solitons and Fractals, vol. 41, pp.1167-1173.

Hanias, M. P.; Giannis, L. I. \& Tombras, G. S. (2010). Chaotic operation by a single transistor circuit in the reverse active region, Chaos, vol. 20, pp. 0131051-0131057.

Holmes, P. (1979). A nonlinear oscillator with a strange attractor, Phil. Trans. Roy. Soc. London A, vol. 292, pp. 419-448.

Kennel M.B.; Brown R. \& Abarbanel H.D.I. (1992). Determining embedding dimension for phase-space reconstruction using a geometrical construction, Phys. Rev. A, vol. 45, pp. 3403-34011.

Lai,Y-C.; Kandangath,A.; Krishnamoorthy,S.; Gaudet, J.A \& de Moura, P.S. (2005). Inducing chaos by resonant perturbations: theory and experiment, Phys. Rev. Lett., vol. 94, pp. 214101-4.

Rizomiliotis, P.; Bogris, A. \& Syvridis, D. (2010). Message Origin Authentication and Integrity Protection in Chaos-Based Optical Communication, IEEE Journal of Quantum electronics, vol. 46, no. 3, pp. 377-383.

Romeira,B.; Figueiredo, J.M. L.; Ironside,C.N. \& Slight,T. (2009). Chaotic Dynamics in Resonant Tunnelling Optoelectronic Voltage Controlled Oscillators, IEEE Photonics Technology Letters, vol. 21, no. 24, pp.1819-1821.

Rosenstein, M.T.; Collins, J.J. \& Deluca C.J. (1993). A practical method for calculating largest Lyapunovexponents, Physica D, vol. 65, pp.117-134.

Stelter, P \& Pfingsten, T. (1991). Calculation of the fractal dimension via the correlation integral, Chaos Solitons $\mathcal{E}$ Fractals, vol 1, iss. 3, pp. 273-280.

Takens, F. (1980). Detecting strange attractors in turbulence, Dynamic Systems and Turbulence, Warwick, 1980, Lecture Notes in Mathematics, vol. 898, edited by D. A. Rand and L. S. Young_Springer, Berlin, 1981, pp. 366-381.

Tamaseviciute, E.; Tamasevicius, A.; Mykolaitis, G., Bumeliene,S. \& Lindberg, E. (2008). Analogue Electrical Circuit for Simulation of the Duffing-Holmes Equation, Nonlinear Analysis: Modelling and Control, vol. 13, no. 2, pp. 241-252. 


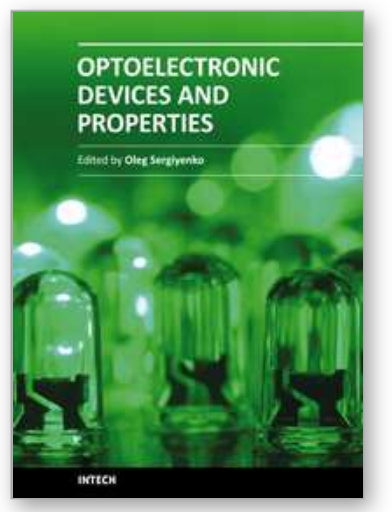

\section{Optoelectronic Devices and Properties}

Edited by Prof. Oleg Sergiyenko

ISBN 978-953-307-204-3

Hard cover, 660 pages

Publisher InTech

Published online 19, April, 2011

Published in print edition April, 2011

Optoelectronic devices impact many areas of society, from simple household appliances and multimedia systems to communications, computing, spatial scanning, optical monitoring, 3D measurements and medical instruments. This is the most complete book about optoelectromechanic systems and semiconductor optoelectronic devices; it provides an accessible, well-organized overview of optoelectronic devices and properties that emphasizes basic principles.

\section{How to reference}

In order to correctly reference this scholarly work, feel free to copy and paste the following:

M.P. Hanias, H.E. Nistazakis and G.S. Tombras (2011). Optoelectronic Chaotic Circuits, Optoelectronic Devices and Properties, Prof. Oleg Sergiyenko (Ed.), ISBN: 978-953-307-204-3, InTech, Available from: http://www.intechopen.com/books/optoelectronic-devices-and-properties/optoelectronic-chaotic-circuits

\section{INTECH}

open science | open minds

\author{
InTech Europe \\ University Campus STeP Ri \\ Slavka Krautzeka 83/A \\ 51000 Rijeka, Croatia \\ Phone: +385 (51) 770447 \\ Fax: +385 (51) 686166 \\ www.intechopen.com
}

\author{
InTech China \\ Unit 405, Office Block, Hotel Equatorial Shanghai \\ No.65, Yan An Road (West), Shanghai, 200040, China \\ 中国上海市延安西路65号上海国际贵都大饭店办公楼405单元 \\ Phone: +86-21-62489820 \\ Fax: $+86-21-62489821$
}


(C) 2011 The Author(s). Licensee IntechOpen. This chapter is distributed under the terms of the Creative Commons Attribution-NonCommercialShareAlike-3.0 License, which permits use, distribution and reproduction for non-commercial purposes, provided the original is properly cited and derivative works building on this content are distributed under the same license. 